\title{
Off-resonant quantum dot-cavity interaction
}

\author{
Arka Majumdar ${ }^{1}$, Erik Kim ${ }^{1}$, Yiyang Gong ${ }^{1}$, Andrei Faraon ${ }^{2}$, Dirk Englund ${ }^{3}$, Jelena Vuckovic ${ }^{1}$ \\ ${ }^{1}$ E. L. Ginzton Laboratory, Stanford University, Stanford, CA-94305; ${ }^{2}$ HP labs, Palo Alto $;{ }^{3}$ Columbia University, New York, USA \\ e-mail: arkam@stanford.edu
}

\begin{abstract}
Off -resonant quantum dot -cavity coupling is studied both experimentally and theoretically. A theoretical model is proposed to explain the observations.

(C)2011 Optical Society of America

OCIS codes:_(230.5590) Quantum-well, -wire and -dot devices;(270.5580) Quantum Electrodynamics
\end{abstract}

Recent demonstrations of cavity quantum electrodynamics (CQED) with a single quantum dot (QD) coupled to a semiconductor micro-cavity show the great potential of this system for developing robust, scalable quantum information processing devices. However, unlike ultra-cold atoms, QDs constantly interact with their local environments and this interaction plays a significant role in CQED experiments. For example, several experiments have reported the observation of cavity emission even when the QD is far detuned ( 3-10 meV) from the cavity resonance, in contrast with atomic CQED experiments. This unexpected non-resonant QD-cavity coupling is observed both in photoluminescence, where the QD is excited by creating carriers above the band-gap of the GaAs surrounding the QD and in the cavity luminescence under resonant excitation of the QD [1]. Figure 1 shows a typical spectrum observed in an off-resonantly coupled QD-cavity system, where we observe emission from the cavity, when the quantum dot is resonantly excited.

Recent theoretical investigations have attributed the off-resonant coupling to several different causes including pure dephasing, phonon relaxation, multi-exciton complexes and charges surrounding the QD. However, to isolate the role of phonons in off-resonant QD-cavity coupling, studies employing resonant excitation of the QD are preferable as they avoid possible complications arising from multi-excitonic complexes and nearby charges generated in above band pumping.

We experimentally study the process responsible for transferring photons between the QD and the off-resonant cavity mode, under resonant excitation of the QD. We observe the power broadening of the QD linewidth and the saturation of the cavity intensity with excitation laser power by monitoring the off-resonant cavity emission (Figure 2) [2]. We fit the saturation data simultaneously with the power broadened line-width, and find that there is an additional power independent broadening of the QD line-width, which we attribute to charge induced spectral diffusion.
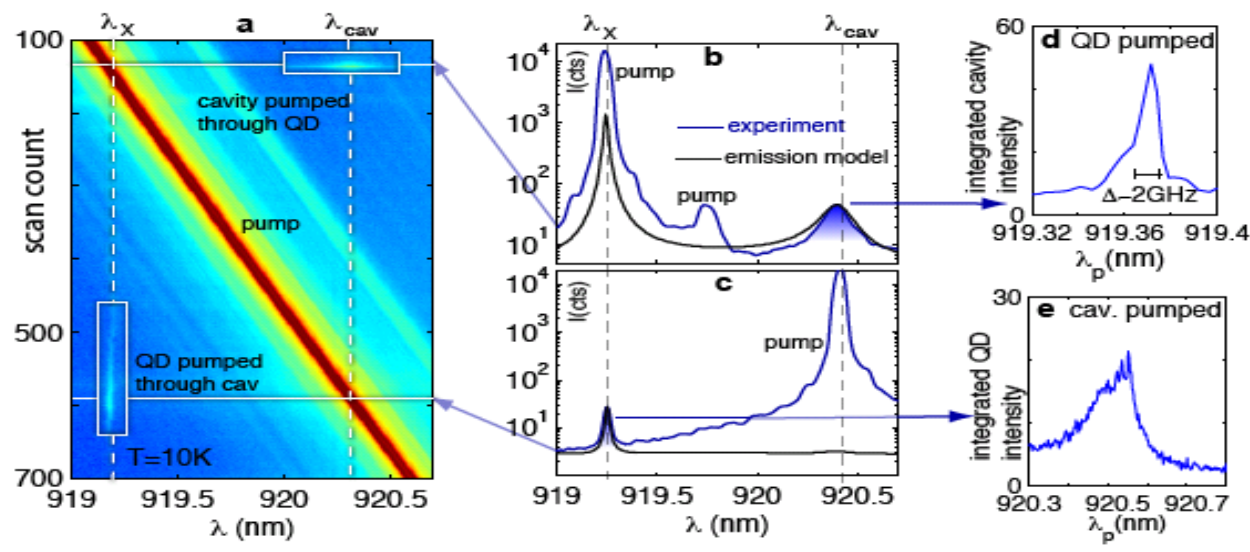

Figure 1: Coherent QD spectroscopy via cavity mode. (a) Intensity on spectrometer when the pump wavelength $\lambda_{\mathrm{p}}$ is scanned across the detuned $\mathrm{QD} /$ cavity system at $10 \mathrm{~K}$. When $\lambda_{\mathrm{p}}$ is resonant with the dot (cavity), we observe emission from the cavity. (b) Spectrum when QD is pumped (the middle peak corresponds to the laser side mode) (c) Spectrum when cavity is pumped. (d) The integrated cavity emission as a function of the pump wavelength $\lambda_{\mathrm{p}}$ shows the single exciton absorption resolved to $3 \mathrm{GHz}$. (e) The integrated QD emission as a function of the pump wavelength $\lambda_{\mathrm{p}}$

We also theoretically model the off-resonant dot-cavity coupling under resonant excitation of the QD. We first use a simple pure dephasing process to model the coupling. However, the phenomenological dephasing model does not 


\section{QThR3.pdf}

explain the complete physics of the coupling. Hence we introduce a new incoherent coupling channel (with rate $\gamma_{\mathrm{r}}$ ) between the cavity and the quantum dot to explain the off-resonant coupling. We find that the new coupling mechanism works for a much larger cavity-QD detuning, as opposed to the pure dephasing model.

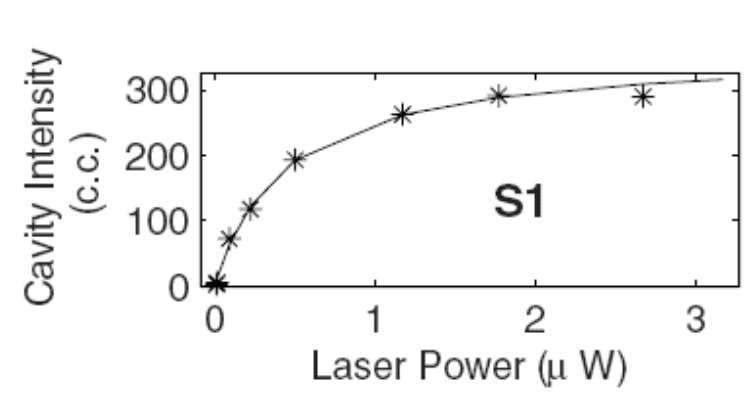

(a)

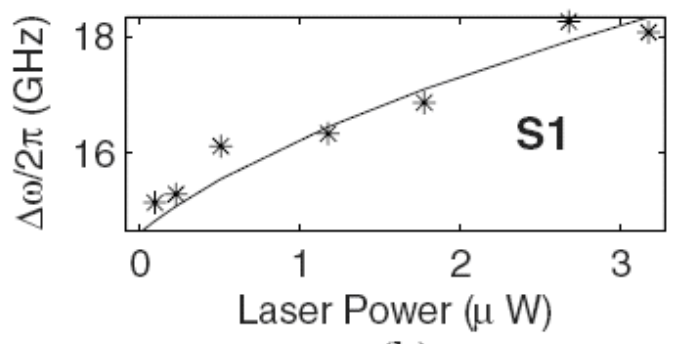

(b)
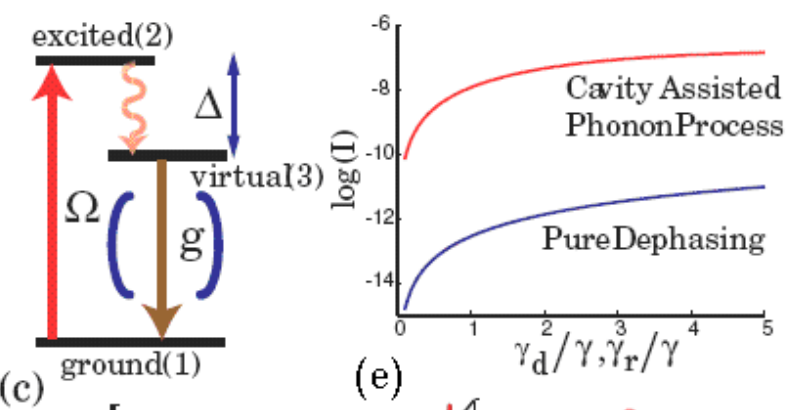

(c)

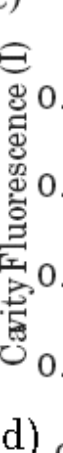

(e)

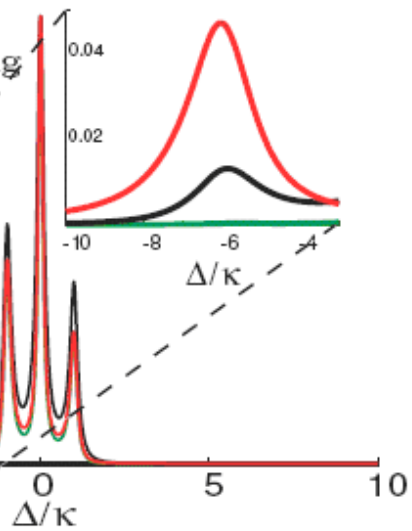

Figure 2: (a) The cavity emission saturation as a function of the laser power. The laser drives an off-resonant quantum dot. (b) The quantum dot linewidth broadens as a function of the laser power. The theoretical fit is obtained by the saturation fit of the cavity emission, and shows that there is a large power independent linewidth. (c) The energy level diagram for the off-resonantly coupled cavity and the QD. The level (2) corresponds to excited QD state, and the level (3) to cavity resonance. In our model, we assume that this transition is phonon-mediated. (d) Theoretical resonance fluorescence spectra obtained from cavity, under the resonant excitation of the QD (level scheme shown in (c)). We observe the Mollow triplet, as we expect from a single QD. Without the introduction of pure dephasing, or cavity enhanced phonon-mediated processes in the theoretical model, we do not observe any off-resonant cavity emission. (e) The cavity emission as a function of the pure dephasing rate $\gamma_{\mathrm{d}}$ and rate $\gamma_{\mathrm{r}}$ which describes the phonon mediated transition between levels (2) and (3). The latter model reproduces much stronger off-resonant cavity output, as observed in the experiments.

Financial support was provided by Army Research Office, Presidential Early Career Award for Engineers and Scientists (PECASE), and SGF (T.I. Fellowship).

\section{References:}

[1] Englund et. al.; Phys. Rev. Lett. 104, 073904 (2010).

[2] Majumdar et. al. ; Phys. Rev. B, 82, 045306, (2010). 